\title{
Novel porous fiber based on dual-asymmetry for low-loss polarization maintaining $\mathrm{THz}$ wave guidance
}

\author{
Islam, Raonaqul; Habib, Selim; Hasanuzzaman, G.K.M.; Rana, Sohel; Sadath, Anwar
}

Published in:

Optics Letters

Link to article, DOI:

10.1364/OL.41.000440

Publication date:

2016

Document Version

Peer reviewed version

Link back to DTU Orbit

Citation (APA):

Islam, R., Habib, S., Hasanuzzaman, G. K. M., Rana, S., \& Sadath, A. (2016). Novel porous fiber based on dualasymmetry for low-loss polarization maintaining THz wave guidance. Optics Letters, 41(3), 440-443.

https://doi.org/10.1364/OL.41.000440

\section{General rights}

Copyright and moral rights for the publications made accessible in the public portal are retained by the authors and/or other copyright owners and it is a condition of accessing publications that users recognise and abide by the legal requirements associated with these rights.

- Users may download and print one copy of any publication from the public portal for the purpose of private study or research.

- You may not further distribute the material or use it for any profit-making activity or commercial gain

- You may freely distribute the URL identifying the publication in the public portal 


\title{
A Porous Fiber Based on Dual-Asymmetry for Low- Loss Polarization Maintaining THz Wave Guidance
}

\author{
RaOnaqul Islam, ${ }^{1}$ Md. Selim Habib, ${ }^{1,2, *}$ G. K. M. Hasanuzzaman, ${ }^{1}$ Sohel \\ RANA, $^{1}$ AND MD. ANWAR SADATH ${ }^{1}$ \\ ${ }^{1}$ Department of Electrical and Electronic Engineering, Rajshahi University of Engineering \& Technology, Bangladesh \\ ${ }^{2}$ DTU Fotonik, Department of Photonics Engineering, Technical University of Denmark, Denmark \\ *Corresponding author: seha@fotonik.dtu.dk
}

Received XX Month XXXX; revisedXX Month, XXXX; accepted XX MonthXXXX; posted XX Month XXXX (Doc. ID XXXXX); published XX Month XXXX

In this paper, we suggest a novel kind of (to our knowledge) porous-core photonic crystal fiber (PCF) for efficient transportation of polarization maintaining (PM) terahertz (THz) waves. We introduce an asymmetry in both the porous-core and the porous-cladding of the structure in order to achieve an ultra-high birefringence. Besides, only circular air-holes have been used to represent the structure, which makes the fiber remarkably simple. The transmission characteristics have been numerically examined based on an efficient finite element method (FEM). The numerical results confirm a high-birefringence of $\sim 0.045$ and a very low effective absorption loss of $0.08 \mathrm{~cm}^{-1}$ for optimal design parameters at 1 THz. We have also thoroughly investigated some important modal properties such as bending loss, power fraction, dispersion, and fabrication possibilities to completely analyze the structure's usability in multitude of $\mathrm{THz}$ appliances. Moreover, physical insights of the proposed fiber have also been discussed. (C) 2015 Optical Society of America

OCIS codes:

http://dx.doi.org/10.1364/OL.99.099999

Artificially induced high-birefringence is effective for polarization maintaining (PM) sensing applications [1]. Recently, highly birefringent waveguides for $\mathrm{THz}$ waves $\left(10^{11}-10^{13} \mathrm{~Hz}\right)$ are also being admired owing their implementation in polarization sensitive $\mathrm{THz}$ time-domain spectroscopy $[2,3]$. These waveguides maintain low absorption co-efficient since material absorption loss for $\mathrm{THz}$ waves is excessively high in existing dielectrics. Most often, majority fraction of the mode power is propagated in dry air to reduce the effective absorption loss, because dry air is assumed as a transparent medium for THz waves.

There had been very few investigations on $\mathrm{THz}$ polarization maintenance, which include plastic PCFs [4], air-core band-gap fibers [5], porous fibers [6-11], elliptical dielectric coated metallic hollow fibers [12], and polymer tubes [13]. The plastic PCF [4] showed a high birefringence (0.021) but an undesirably high transmission loss $(400 \mathrm{~dB} / \mathrm{m}$ at $1 \mathrm{THz})$. Later, the solid-core of the plastic PCF was replaced with an air-core [5] to reduce the fiber propagation loss; which, however, resulted in a lower birefringence of the order $10^{-3}$. Meanwhile, there were reports on squeezed lattice elliptical-hole structures [6] and super-cell structures [7] which showed birefringence of the order 10-2. Excellent improvements were made by porous fibers with rectangular slots $[8,9]$ and porous fibers with rotated elliptical air-holes [10] that showed high birefringence of $\sim 0.026$ and ultra-high birefringence of $\sim 0.045$ respectively. In 2015, authors in [11] presented a slotted porous-core design that exhibited an extremely high-birefringence of $\sim 0.075$. Metallic hollow fiber with dielectric coating [12] was also admirable since it showed high modal birefringence $\left(\sim 1.36 \times 10^{-2}\right)$ and propagated maximum power through an air-core to exhibit very low effective absorption loss $\left(\sim 0.003 \mathrm{~cm}^{-1}\right)$. Nevertheless, all these structures described in Refs. [6-12] suffer from the fact that they are difficult to be realized practically due to their complicated shapes and patterns. Polymer tubes [13] are advantageous for their high-birefringence and low-loss property, but they are subjected to be infiltrated with uncertain atmospheric losses.

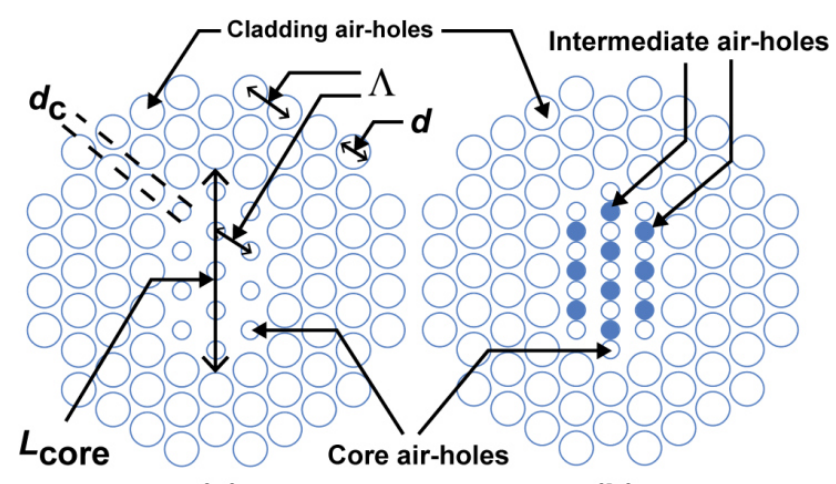

(a)

(b)

Fig. 1. Schematic diagram of the proposed design with the symmetry broken in a) the cladding, and b) both in the cladding and the core. The solid blue circles in (b) indicate the intermediate air-holes. 
In the previous literatures, the high-birefringence in PCFs has thus far been achieved by breaking the symmetry of either the cladding [4-7] or the core [8-11]. In this work, we suggest a novel idea where symmetry is broken in both the core and the cladding. We call this scheme as the dual asymmetry. This proposed unique structure gives tremendous flexibility during fabrication because only circular airholes have been used.

Figure 1 shows a complete cross-section of the proposed porouscore PCF. It can be observed from Fig. 1 that the proposed design is based on regular hexagonal lattice structure with five rings of air-holes. In Fig. 1(a), it is shown that an asymmetric cladding is formed by reducing the diameter $(d)$ of some specific air-holes to a lower diameter $\left(d_{c}\right)$. The smaller air-holes with diameter $d_{c}$ are called the core air-holes, $L$ core indicates the length of the porous-core, and the pitch denoted by $\Lambda$, is called the distance between two adjacent airholes. The pitch is kept fixed for both the core and the cladding. The core air -filling ratio $\left(d_{c} / \Lambda\right)$ is varied to change the diameter of the core air-holes. In the proposed design, an intermediate air-hole is placed in the gaps between two vertically adjacent core air-holes in order to break the symmetry of the core (see Fig. 1(b)). The diameter of the intermediate air-holes (filled with blue circles) is chosen similar to the diameter of the original core air-holes of Fig. 1(a) in order to reduce fabrication difficulties. Each group of the three nearby intermediate airholes create a triangular lattice, and the distance between any two intermediate air-holes of that lattice is equal to the pitch $(\Lambda)$ of the fiber. Please note that the radius of the total fiber is $405 \mu \mathrm{m}$ when the core length ( $\left.L_{\text {core }}\right)$ is $400 \mu \mathrm{m}$. The background material chosen for this design is Cyclic Olefin Copolymer (trade name- TOPAS) for its excellent properties such as constant refractive index $(n=1.53)$ between $0.1-2$ $\mathrm{THz}$ [14], low bulk material loss of $0.2 \mathrm{~cm}^{-1}$ at $1 \mathrm{THz}$ [15], insensitivity to humidity [16], decency for bio-sensing [17], and the fact that it can be made from different grades to achieve high glass transition temperature [18]. The numerical simulations are performed using commercially available FEM based state-of-the-art COMSOL software. A circular perfectly matched layer (PML) is applied outside the computational domain in order to calculate the loss. The FEM computed power flow distributions at various core lengths are shown in Fig. 2, where it is observed that mode power is tightly confined around the asymmetric porous-core, which is essential in THz wave guidance.

In order to examine the performance of the proposed fiber as a polarization maintaining $\mathrm{THz}$ waveguide, we first analyze the birefringence property. The birefringence is expressed as [10]

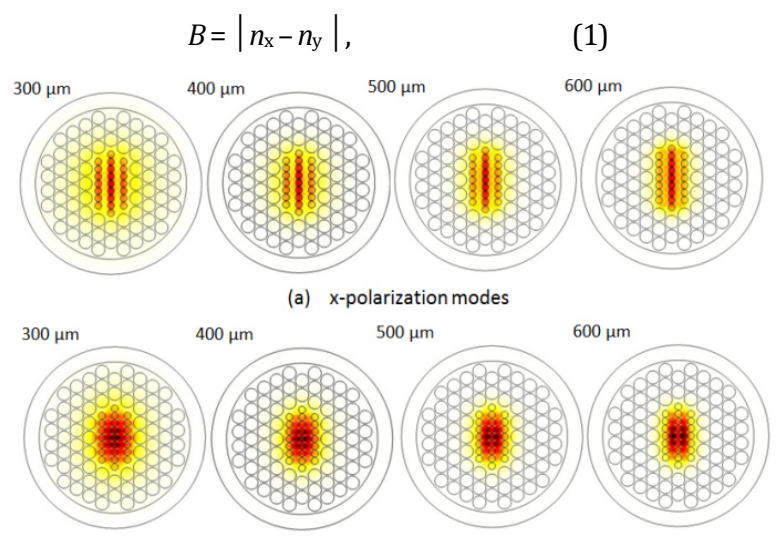

(b) $y$-polarization modes

Fig. 2. Power flow distribution of the proposed fiber a) $x$-polarization modes and b) $y$-polarization modes for various core lengths and $d_{c} / \Lambda=$ 0.48 . where $B$ stands for birefringence, $n_{\mathrm{x}}$ and $n_{\mathrm{y}}$ indicate $x$ and $y$ polarization modes of the effective refractive index respectively. First, the birefringence is examined at $1 \mathrm{THz}$ for different core air -filling ratio. The results are shown in Fig. 3 in which it is observed that the birefringence is increased when $d_{c} / \Lambda$ is increased for a constant core length. Note that the increment rate is higher for larger core lengths (Fig. 3). Interestingly, we noticed in Fig. 3 that for $d_{c} / \Lambda \lesssim 0.37$, the birefringence is higher for lower core length, and for $d_{c} / \Lambda \gtrsim 0.37$, the birefringence is higher for higher core length. To understand this mechanism, we first consider the situation when the symmetry of the cladding is broken. At that point, if $L_{\text {core }}$ is increased, the asymmetry ratio (length of mode field along $y$-axis divided by its length along $x$ axis) is decreased. This occurrence reduces the overall birefringence of the fiber. On the other hand, if $L_{\text {core }}$ is increased when the symmetry of the core is broken, the core air-holes and intermediate air-holes are enlarged. Therefore the asymmetry ratio is rapidly increased which consequently enhances the birefringence. Obviously, high birefringence over 0.04 is obtained at $f=1 \mathrm{THz}, d_{c} / \Lambda=0.48, L_{\text {core }}=400$ $\mu \mathrm{m}, 500 \mu \mathrm{m}$, and $600 \mu \mathrm{m}$ (see Fig. 3). This result is very much comparable to the previously reported PM THz fibers [4-13]. Next, Fig. 4 shows the frequency dependency of birefringence of the proposed fiber. It is observed from Fig. 4 that the bandwidth of birefringence increases with core length. For example, for $L_{\text {core }}=600 \mu \mathrm{m}$, birefringence is higher than 0.04 within $0.6-2 \mathrm{THz}$ frequency band, whereas for $L_{\text {core }}=400 \mu \mathrm{m}$, the birefringence stays above 0.04 within a narrower band of $0.95-2 \mathrm{THz}$. This is due to the fact that under dual asymmetry, a higher core length shows higher birefringence at the same frequency. Therefore, $L_{\text {core }}=600 \mu \mathrm{m}$ reaches 0.04 far before $L_{\text {core }}$ $=400 \mu \mathrm{m}$. It is necessary to mention that no previous report on PM THz fibers showed high birefringence for such wide bands [4-13].

We now study one of the most important modal properties of $\mathrm{THz}$ fibers, material absorption loss. The effective absorption loss is quantified as [10]

$$
\alpha_{\text {eff }}=\sqrt{\frac{\varepsilon_{0}}{\mu_{0}}}\left(\frac{\int_{\text {mat }} \mathrm{n}_{\text {mat }}|\mathrm{E}|^{2} \alpha_{\mathrm{mat}} \mathrm{dA}}{\left|\int_{\mathrm{all}} \mathrm{S}_{\mathrm{z}} \mathrm{dA}\right|}\right),
$$

where $\varepsilon_{0}$ and $\mu_{0}$ are the permittivity and permeability of the vacuum, $n_{\text {mat }}$ is the refractive index of the material used, $E$ is the modal electric field, $\alpha_{\text {mat }}$ is the bulk material absorption loss and $S_{\mathrm{z}}$ is the $z$ component of the Poynting vector. Material absorption loss in the proposed fiber can be understood from Fig. 5, where it is observed that the effective absorption loss is reduced if either $d_{c} / \Lambda$ is increased or $L_{\text {core }}$ is reduced. However, $d_{c} / \Lambda$ cannot be extended above 0.48 because, at that point, core air-holes overlap the intermediate air-holes which results in an impractical fiber design. Note that the effective absorption loss for $L_{\text {core }}=400 \mu \mathrm{m}$, and $d_{c} / \Lambda=0.48$ is $0.08 \mathrm{~cm}^{-1}$ for the $x$ polarized mode and $0.11 \mathrm{~cm}^{-1}$ for the $y$-polarized mode of the guided wave.

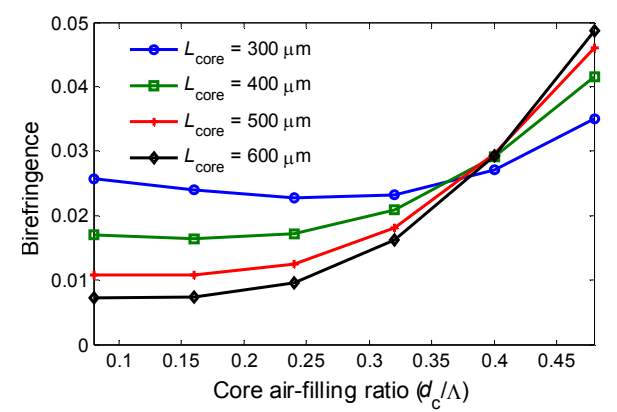

Fig. 3. Birefringence versus the air filling ratio of the core for different core lengths at $1 \mathrm{THz}$. 


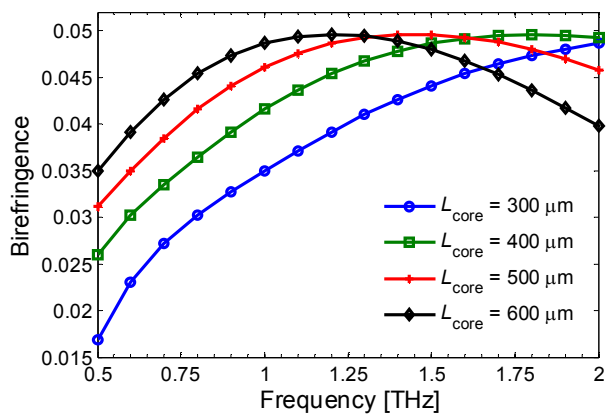

Fig. 4. Birefringence versus frequency at $d_{c} / \Lambda=0.48$ for different core lengths.

This reported absorption loss is lower than most of the previously reported highly birefringent $\mathrm{THz}$ designs $[4,6-10,13]$. It has been acknowledged from a previous report that the bulk material loss of TOPAS is $0.06 \mathrm{~cm}^{-1}$ at $0.4 \mathrm{THz}$ and increases at a rate of $0.36 / \mathrm{THz}$ [8]. Bearing this in mind, we calculated effective material loss of the proposed fiber as a function of frequency which is shown in Fig. 6. Figure 6 shows that the loss increases steeply with frequency and the $y$-polarized mode shows higher loss than the $x$-polarized mode. The physical reason is due to the fact that more power propagates through the materials for $y$-polarized waves than $x$-polarized waves which are clearly shown in Fig. 7.

In order to obtain an ultra-high birefringence and simultaneously avoid the effects of material absorption in the THz region, the majority of power should be concentrated in core air-holes. The fraction of mode power in any desirable area can be calculated using [10]

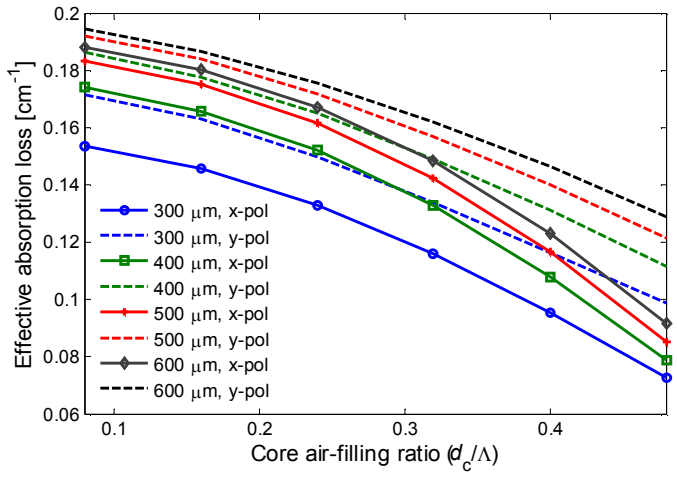

Fig. 5. Effective material absorption loss of the proposed fiber as a function of core air filling ration for different core lengths at $1 \mathrm{THz}$.

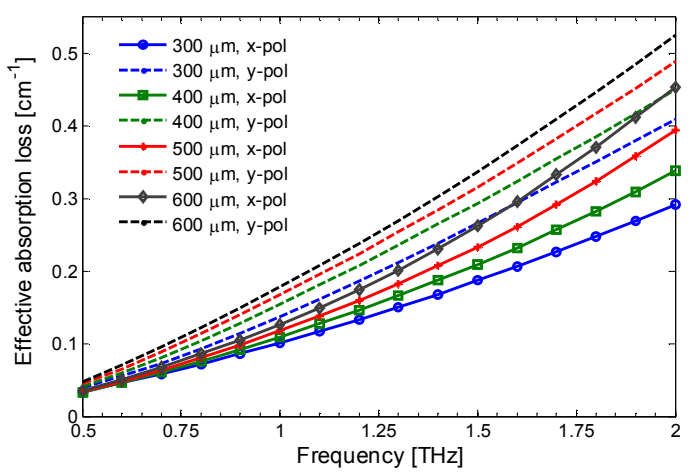

Fig. 6. Effective material absorption loss of the proposed fiber as a function of frequency at core $d_{c} / \Lambda=0.48$ for different core lengths.

$$
\eta^{\prime}=\frac{\int_{X} S_{z} d A}{\int_{\text {all }} S_{z} d A^{\prime}}
$$

where the integration of the denominator is done for all areas over the fiber's cross-section and the numerator for the area covered by core air-holes (denoted by $X$ ). The power fraction in core air is shown as a function of $d_{c} / \Lambda$ in Fig. $8 \mathrm{in}$ which it is observed that power fraction in the core air-holes is increased if either $d_{c} / \Lambda$ or core length is increased. Additionally, Fig. 9 shows that maximum power fraction in core air (around $40 \%$ of the total power) is achieved at lower frequencies for higher core lengths. It was found that power fraction in core air is $33 \%$ of the total power for $x$-polarized mode and $31 \%$ for $y$ polarized mode for $L_{\text {core }}=400 \mu \mathrm{m}$, and $d_{c} / \Lambda=0.48$ at $f=1 \mathrm{THz}$.

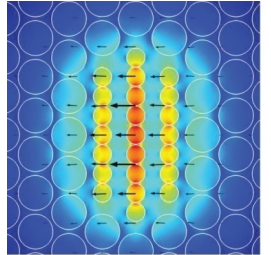

(a)

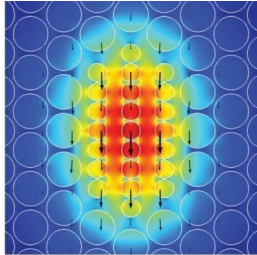

(b)
Fig. 7. Distribution of the electric field of the normalized power-flow at $L_{\text {core }}=400 \mu \mathrm{m}, f=1 \mathrm{THz}$, and $d / \Lambda=0.48$ for a) $x$-polarized mode and b) $y$-polarized mode. The black arrows show the directions of the electric field.

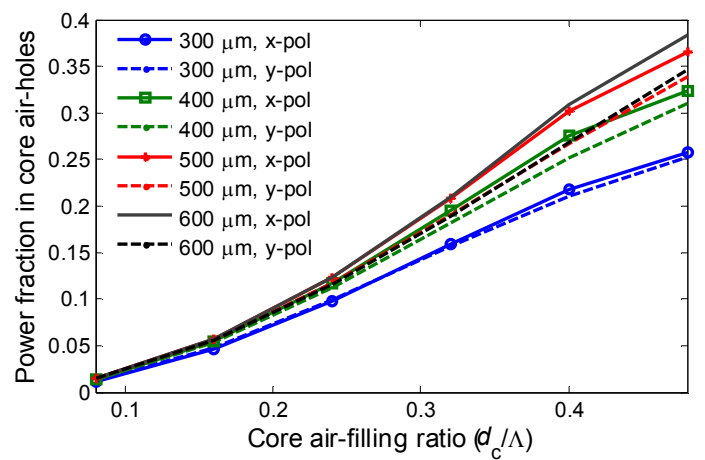

Fig. 8. Fraction of mode power in core air-holes versus core air filling fraction at $1 \mathrm{THz}$ for different core lengths.

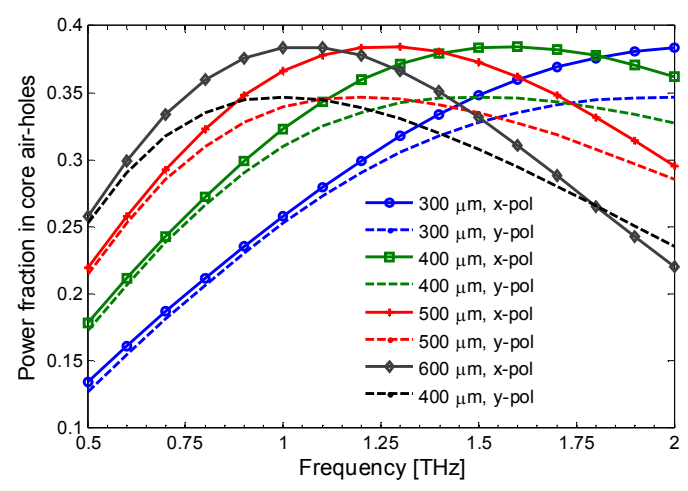

Fig. 9. Fraction of mode power in core air-holes of the proposed waveguide as a function of frequency at core $d_{c} / \Lambda=0.48$ for different core lengths. 


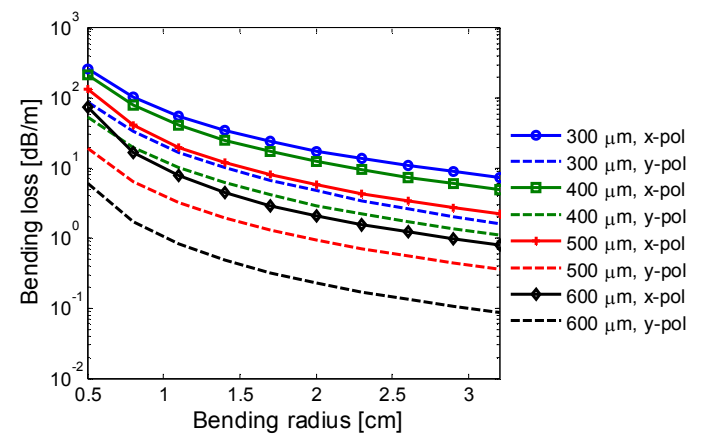

Fig. 10. Bending loss of the proposed fiber as a function of bending radius at $1 \mathrm{THz}$, core $d_{c} / \Lambda=0.48$ for different core lengths.

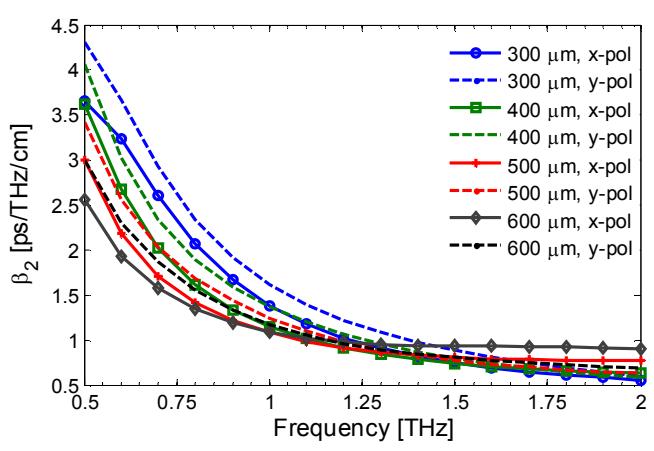

Fig. 11. Dispersion characteristics of the proposed waveguide as a function of frequency at core $d_{c} / \Lambda=0.48$ for different core lengths.

Next, to calculate bending loss, the bent fiber is replaced with its equivalent straight fiber and the effective refractive index of that fiber is used to calculate the leakage loss. The equivalent refractive index can be found by using the conformal transformation method [19] and the modified equivalent index distribution can be expressed as [19]

$$
n_{\mathrm{eq}}(x, y)=n(x, y) \exp (1+x / R)
$$

where, $n(x y)$ is the original refractive index profile of the fiber, $R$ is the bending radius and $x$ is the distance from the center of the fiber. Figure 10 shows the bending loss of the proposed PCF at various bending radii. It is observed from Fig. 10 that the loss is reduced when either bending radius or core length is increased. This is obvious since both the higher bending radius and larger core length allow more area for the guided mode of the bent fiber. It is also observed from Fig. 10 that $y$-polarized mode shows lower loss than $x$-polarized mode, which is due to the fact that $x$-polarized mode is more delocalized from the center than the $y$-polarized mode for bending along the $x$-axis.

To investigate the dispersion properties, we consider only the waveguide dispersion because TOPAS has near zero material dispersion in between 0.1-2 THz [14]. The waveguide dispersion is realized using the dispersion parameter, $\beta_{2}$, expressed as [20]

$$
\beta_{2}=\frac{2}{c} \frac{d n_{\text {eff }}}{d \omega}+\frac{\omega}{c} \frac{d^{2} n_{\text {eff }}}{d \omega^{2}}
$$

where $\omega=2 \pi f$ and $c\left(=3 \times 10^{8} \mathrm{~ms}^{-1}\right)$ is the velocity of light in vacuum. Figure 11 shows the dispersion as a function of frequency at various core lengths. It is observed from Fig. 11 that at frequencies lower than $1 \mathrm{THz}$, a lower core length of the fiber results in higher dispersion. However, at frequencies higher than $1 \mathrm{THz}$, dispersion is nearly flat for any fiber dimensions proposed in this work. Furthermore, the $x$ polarized mode shows lower dispersion than the $y$-polarized mode in the whole band of interest $(0.5-2 \mathrm{THz})$. This is due to the fact that the power is more confined inside the core for $x$-polarized modes than $y$ polarized modes. Note that for $L_{\text {core }}=400 \mu \mathrm{m}$ and $d_{c} / \Lambda=0.48$, the dispersion is $0.9 \pm 0.26 \mathrm{ps} / \mathrm{THz} / \mathrm{cm}$ for $x$-polarized mode and $0.98 \pm$ $0.39 \mathrm{ps} / \mathrm{THz} / \mathrm{cm}$ for $y$-polarized mode of the guided wave.

It is well known that stacking, extrusion and drilling are the methods of forming PCFs from polymer and air [21]. In 2004, Barton et al. described a drilling and drawing technique [22] to fabricate polymer fibers. The porous-core honeycomb fiber proposed in [23] is also fabricated [14]. It is clear from Fig. 1 that the proposed fiber only contains circularly shaped air-holes and a typical hexagonal structure. Therefore, it is logical to anticipate that the dually asymmetric fiber can easily be fabricated using the verified methods of polymer fiber fabrication.

In conclusion, we have explored a new kind of porous-core PCF which introduces dual asymmetry using intermediate air-holes and an asymmetric cladding. This relatively simple structure has been realized using only circular air-holes and the estimated size of the fiber is comparatively small. The key attributes of the proposed fiber are its simple design, easier manufacturability, ultra-high birefringence and very low effective material absorption loss. Thus, we can expect of this fiber to be a noteworthy contribution to the $\mathrm{THz}$ polarization maintaining applications.

Acknowledgment. The authors acknowledge fruitful discussion with Professor Ole Bang.

\section{References}

1. J. Noda, K. Okamoto, and Y. Sasaki, J. Lightwave Technol. 4, 1071(1986).

2. M. B. Byrne, M. U. Shaukat, J. E. Cunningham, E. H. Linfield, and A. G. Davies, Appl. Phys. Lett. 98, 151104 (2011).

3. N. Karpowicz, et al., Appl. Phys. Lett. 92, 011131 (2008).

4. M. Cho, J. Kim, H. Park, Y. Han, K. Moon, E. Jung, and H. Han, Opt. Express 16, 7 (2008).

5. G.-B. Ren, Y.-D. Gong, P. Shum, X. Yu, and J.-J. Hu, IEEE J. Quantum Electron. 45, 506 (2009).

6. H.-B. Chen, D.-R. Chen, and Z. Hong, Appl. Opt. 48, 3943 (2009).

7. D.-R. Chen and H. Y. Tam, J. Lightwave Technol., 28, 1858 (2010).

8. S. Atakaramians, S. Afshar Vahid, B. M. Fischer, D. Abbott, and T. M. Monro, Opt. Commun. 282, 36 (2009).

9. S. Atakaramians, S. Afshar V, H. Ebendorff-Heidepriem, M. Nagel, B. M. Fischer, D. Abbott, and T. M. Monro, Opt. Express 17, 14053 (2009).

10. N. Chen, J. Liangand, L. Ren, Appl. Opt. 52, 5297 (2013).

11. R. Islam, M. S. Habib, G. K. M. Hasanuzzaman, R. Ahmad, S. Rana, and S. F. Kaijage, IEEE Photon. Technol. Lett. 27, 2222, (2015).

12. Xiao-Li Tang, Bang-Shan Sun, and Yi-Wei Shi, Opt. Express, 19, 24967 (2011).

13. D.-R. Chen, J. Lightwave Technol. 28, 2708 (2010).

14. H. Bao, K. Nielsen, H. K. Rasmussen and P. U. Jepsen, Opt. Express 20, 29507 (2012)

15. K. Nielsen, H. K. Rasmussen, A. J. Adam, P. C. Planken, O. Bang, and P. U. Jepsen, Opt. Exp. 17, 8592 (2009).

16. W. Yuan, L. Khan, D. J. Webb, K. Kalli, H. K. Rasmussen, A. Stefani, O. Bang, Opt. Express 19, 19731 (2011).

17. G. Emiliyanov, P. E. Høiby, L. H. Pedersen and O. Bang, Sensors 13, 3242 (2013).

18. C. Markos, A. Stefani, K. Nielsen, H. K. Rasmussen, W. Yuan and O. Bang, Opt. Express 21, 4758 (2013).

19. M. Heiblum, J. Harris, IEEE J. Quantum Electron. 11, 75 (1975).

20. J. Liang, L. Ren, N, Chen, C. Zhou, Opt. Commun. 295, 257 (2013).

21. M. A. van Eijkelenborg, et al., Opt. Express 9, 319 (2001).

22. G. Barton, M. A. van Eijkelenborg, G. Henry, M. C.J. Large, J. Zagari, Opt. Fiber Technol. 10, 325 (2004).

23. K. Nielsen, H. K. Rasmussen, P. U. Jepsen, and O. Bang, Opt. let., 36, 5 ( 2011). 


\section{References}

1. J. Noda, K. Okamoto, and Y. Sasaki, "Polarization-maintaining fibers and their applications," J. Lightwave Technol., vol. 4, no. 8, pp. 1071-1089, 1986.

2. M. B. Byrne, M. U. Shaukat, J. E. Cunningham, E. H. Linfield, and A. G. Davies, "Simultaneous measurement of orthogonal components of polarization in a free-space propagating terahertz signal using electro-optic detection," Appl. Phys. Lett., vol. 98, no. 15, pp. 151104, 2011.

3. N. Karpowicz, et al., "Coherent heterodyne time-domain spectrometry covering the entire "terahertz gap"," Appl. Phys. Lett., vol. 92, no. 1, pp. 011131, 2008.

4. M. Cho, J. Kim, H. Park, Y. Han, K. Moon, E. Jung, and H. Han, “Highly birefringent terahertz polarization maintaining plastic photonic crystal fibers," Opt. Express, vol. 16, no. 1, pp. 7-12, 2008.

5. G.-B. Ren, Y.-D. Gong, P. Shum, X. Yu, and J.-J. Hu, "Polarization Maintaining Air-Core Bandgap Fibers for Terahertz Wave Guiding," IEEE J. Quantum Electron., vol. 45, no. 5, pp. 506-513, 2009.

6. H.-B. Chen, D.-R. Chen, and Z. Hong, "Squeezed lattice elliptical-hole terahertz fiber with high birefringence," Appl. Opt., vol. 48, no. 20, pp. 3943-3947, 2009.

7. D.-R. Chen and H. Y. Tam, "Highly birefringent terahertz fibers based on super-cell structures," J. Lightwave Technol., vol. 28, no. 12, pp. 18581863, 2010.

8. S. Atakaramians, S. Afshar Vahid, B. M. Fischer, D. Abbott, and T. M. Monro, "Low loss, low dispersion and highly birefringent terahertz porous fibers," Opt. Commun., vol. 282, no. 1, pp. 36-38, 2009.

9. S. Atakaramians, S. Afshar V, H. Ebendorff-Heidepriem, M. Nagel, B. M. Fischer, D. Abbott, and T. M. Monro, "THz porous fibers: design, fabrication and experimental characterization," Opt. Express, vol. 17, no. 16, pp. 14053-15062, 2009.

10. N. Chen, J. Liangand, L. Ren, “High-birefringence, low-loss porous fiber for single-mode terahertz-wave guidance," Appl. Opt., vol. 52, no. 21, pp. 5297-5302, 2013.

11. R. Islam, M. S. Habib, G. K. M. Hasanuzzaman, R. Ahmad, S. Rana, and S. F. Kaijage, "Extremely high-birefringent asymmetric slotted core photonic crystal fiber in THz regime," IEEE Photon. Technol. Lett., vol. 27, no. 21, pp. 2222-2225, 2015.

12. Xiao-Li Tang, Bang-Shan Sun, and Yi-Wei Shi, "Design and optimization of low-loss high-birefringence hollow fiber at terahertz frequency," Opt. Express, vol. 19, no. 25, pp. 24967-24979, Nov. 2011.

13. D.-R. Chen, "Mode property of terahertz polymer tube," J. Lightwave Technol., vol. 28, no. 18, pp. 2708-2713, 2010.

14. H. Bao, K. Nielsen, H. K. Rasmussen and P. U. Jepsen, "Fabrication and characterization of porous-core honeycomb bandgap THz fibers," Opt. Express, vol. 20, no. 28, pp. 29507-29517, Dec. 2012.

15. K. Nielsen, H. K. Rasmussen, A. J. Adam, P. C. Planken, O. Bang, and P. U. Jepsen, "Bendable, low-loss topas fibers for the terahertz frequency range," Opt. Exp., vol. 17, no. 10, pp. 8592-8601, May 2009.

16. W. Yuan, L. Khan, D. J. Webb, K. Kalli, H. K. Rasmussen, A. Stefani, O. Bang, "Humidity insensitive TOPAS polymer fiber Bragggrating sensor," Opt. Express, vol. 19, no. 20, pp. 19731-19739, Sep. 2011.

17. G. Emiliyanov, P. E. Høiby, L. H. Pedersen and O. Bang, "Selective serial multi-antibody biosensing with TOPAS microstructured polymer optical fibers," Sensors, vol. 13, pp. 3242-3251, Mar. 2013.

18. C. Markos, A. Stefani, K. Nielsen, H. K. Rasmussen, W. Yuan and O. Bang, "High-Tg TOPAS microstructured polymer optical fiber for fiber Bragg grating strain sensing at 110 degrees," Opt. Express, vol. 21, no. 4, pp. 4758-4765, Feb. 2013.

19. M. Heiblum, J. Harris, "Analysis of curved optical waveguides by conformal transformation," IEEE J. Quantum Electron., vol. 11, no. 2, pp. 75-83, 1975.
20. J. Liang, L. Ren, N, Chen, C. Zhou, "Broadband, low-loss, dispersion flattened porous-core photonic bandgap fiber for terahertz $(\mathrm{THz})$-wave propagation," Opt. Commun., vol. 295, pp. 257-261, 2013.

21. M. A. van Eijkelenborg, et al., "Microstructured polymer optical fibre," Opt. Express, vol. 9, no. 7, pp. 319-327, Sep. 2001.

22. G. Barton, M. A. van Eijkelenborg, G. Henry, M. C.J. Large, J. Zagari, "Fabrication of microstructured polymer optical fibres," Opt. Fiber Technol., vol. 10, no. 4, pp. 325-335, 2004.

23. K. Nielsen, H. K. Rasmussen, P. U. Jepsen, and O. Bang, "Porous-core honeycomb bandgap THz fiber", Opt. let., vol. 36., No. 5, 2011. 